\title{
Article
}

\section{Influence of Drift on Robot Repeatability and Its Compensation}

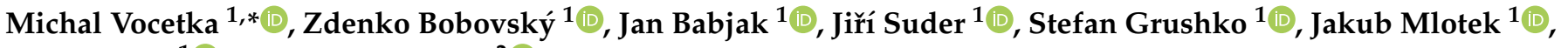 \\ Václav Krys ${ }^{1}$ (D) and Martin Hagara ${ }^{2}$ (D) \\ 1 Department of Robotics, Faculty of Mechanical Engineering, VŠB-Technical University of Ostrava, \\ 70800 Ostrava, Czech Republic; zdenko.bobovsky@vsb.cz (Z.B.); jan.babjak@vsb.cz (J.B.); \\ jiri.suder@vsb.cz (J.S.); stefan.grushko@vsb.cz (S.G.); jakub.mlotek@vsb.cz (J.M.); vaclav.krys@vsb.cz (V.K.) \\ 2 Department of Applied Mechanics and Mechanical Engineering, Faculty of Mechanical Engineering, \\ Technical University of Košice, 04200 Košice, Slovakia; martin.hagara@tuke.sk \\ * Correspondence: michal.vocetka@vsb.cz
}

check for updates

Citation: Vocetka, M.; Bobovský, Z.; Babjak, J.; Suder, J.; Grushko, S.; Mlotek, J.; Krys, V.; Hagara, M. Influence of Drift on Robot Repeatability and Its Compensation. Appl. Sci. 2021, 11, 10813. https:// doi.org/10.3390/app112210813

Academic Editor: Alberto Doria

Received: 12 October 2021

Accepted: 13 November 2021

Published: 16 November 2021

Publisher's Note: MDPI stays neutral with regard to jurisdictional claims in published maps and institutional affiliations.

Copyright: (c) 2021 by the authors. Licensee MDPI, Basel, Switzerland. This article is an open access article distributed under the terms and conditions of the Creative Commons Attribution (CC BY) license (https:// creativecommons.org/licenses/by/ $4.0 /)$.

\begin{abstract}
This paper presents an approach to compensate for the effect of thermal expansion on the structure of an industrial robot and thus to reduce the repeatability difference of the robot in cold and warm conditions. In contrast to previous research in this area that deals with absolute accuracy, this article is focused on determining achievable repeatability. To unify and to increase the robot repeatability, the measurements with highly accurate sensors were performed under different conditions on an industrial robot ABB IRB1200, which was equipped with thermal sensors, mounted on a pre-defined position around joints. The performed measurements allowed to implement a temperature-based prediction model of the end effector positioning error. Subsequent tests have shown that the implemented model used for the error compensation proved to be highly effective. Using the methodology presented in this article, the impact of drift can be reduced by up to $89.9 \%$. A robot upgraded with a compensation principle described in this article does not have to be warmed up as it works with the same low repeatability error in the entire range of the achievable temperatures.
\end{abstract}

Keywords: robot repeatability; robot precision; robot drift; robot warm-up

\section{Introduction}

Contemporary industrial robots are precise machines. For a common industrial angular six-axis robot with a load capacity of up to $10 \mathrm{~kg}$, a repeatability value of 0.025 $\mathrm{mm}$ is achievable. However, this value guaranteed by the manufacturers of those robots is valid only for the steady environment conditions. When the ambient temperature or temperature of the robot changes, the repeatability can drop to significantly worse values with a difference up to one order of magnitude. The value of repeatability is affected by many influences. The greatest one is the drift, caused by thermal expansion. It is the reason why, for many applications, robots must be pre-warmed before production can be started. The phenomenon, generally known as a drift, is defined by ISO 9283:1998 standard [1] as a shift of the position in time.

The thermal expansion and its effect on repeatability is an issue accompanying any high precision device, and thus, there are solutions that describe possible compensation and calibration methods. Among the commercially available solutions, there can be found an AccuBeam: Robot Temperature Compensation system. It uses a twin beam laser and a rugged industrial sensor. Between production cycles, the robot automatically executes a "Quick-Check" measurement routine to assess overall system performance and to detect any drift of Tool Center Point (TCP) or crash of the robot. However, the presence of laser sensors in the workspace is required [2]. For special robot applications, such as honing of boreholes, Wiest AG offers an industrial solution of continuous compensation of the robot temperature drift with the help of the measuring technique LaserLAB [3].

Real-time compensation of $3 \mathrm{DOF}$ ultra-high precision parallel robot, based on the use of a 6 DOF measuring system described in [4] effectively compensates the repeatability 
error. A similar approach is used in real-time $\mathrm{CNC}$ thermal error compensation [5]. One of the approaches to solve the repeatability error is the calibration of the robot for a specific condition. The authors in [6] present a basic overview of robot calibration. Another solution suitable for industrial application is presented in [7] with the use of the finite element theory, which is utilized to create thermal distribution and deformation models of a measured system. A compensation system based on those models can reduce the final position error below $0.1 \mathrm{~mm}$. Robot development centers, as well as university researchers, are working on drift minimization $[8,9]$

To obtain a reliable and robust model, a Grey Neural Network model with Convolution Integral methodology presented by [10] can be used. By using a neural network algorithm and camera-based measurement system, industrial robot calibration is performed in [11] resulting in significant improvement in precision compared to traditional analytical methods. There are other methods of measuring and increasing robot precision. According to [12], the joint errors can be compensated by identifying the joint deformation. With this information, the system can compensate for the predicted deviation caused by the joint stiffness error and the kinematic error by tens of percent. Real-time compensation of thermal error, based on an error distribution model, proposed by [13], can also increase the achievable level of robot precision, as was experimentally verified on a car-body assembly line. The theoretical prediction of the error distribution inside the robot working envelope is realized by using multiple vision sensors. A real-time error compensation can be achieved [14]. The [15] uses an advanced algorithm, such as Kalman filter and MOIFA data fusion, to increase robot precision. By the proposed methodology, an improvement in a range from $38 \%$ to $78 \%$ could be achieved. Another method of robot repeatability measurement is proposed by [16]. In addition to detection and compensation of the 6 DOF position of the robot, it offers an option of quick identification and calibration of the whole workspace (camera subsystem or another periphery, for example) that is based on a pose detection [17].

Highly accurate robotic assembly is sensitive to temperature change. However, in [18], it was presented that by choosing a suitable position inside the robot workspace, a higher probability of assembling the parts correctly can be achieved. Error compensation, based on cokriging is proposed in [19]. The methodology presented in this article is used for an absolute accuracy error compensation and verified in robot drilling applications. The average positional error was reduced from $0.717 \mathrm{~mm}$ to $0.115 \mathrm{~mm}$. Robot machining in the aerospace industry is a challenging task and considering the fact that the requirements for a robot precision are higher than the ordinary robot precision, a real-time pose control and guidance presented in [20] offers a solution. Few studies have presented another way of dealing with the thermal expansion repeatability error. The solution proposed by the [21] is to find an area inside the robot's working envelope in which the influence of thermal expansion on the repeatability is less significant. By defining the movement inside such an area or avoiding the surrounding area that is less suitable for precise movements, a higher level of repeatability could be reached. The differences between the most common robot kinematics, the 6 DOF, SCARA, and gantry structures and their thermal behavior is discussed in [22]. The article includes suggestions for compensation for thermal expansion errors. A simulation of thermal influence on robot structure is described in [23]. A thermally stable material used for the robot body would increase the resistance against the influence of gearbox heat on TCP precision. The theoretical study [24] presents this idea.

The article follows up on our previous research [25], which described the principle of improving the repeatability by changing the robot-to-target approach direction. During experimental measurements performed for this research, we have found that the effect of drift was significant, and a time-consuming preheating of the robot was necessary to neutralize the influence of the changing temperature. Therefore, the question arose whether it was possible to compensate for the drift effect by knowing the actual temperature of the robot structure. 
We, therefore, present a hypothesis: knowing the current temperature of the robot structure, it is possible to compensate for the drift effect and thus eliminate the need to heat the robot to operating temperature before starting.

It is generally accepted that an industrial robot is a universal device; however, when a robot is equipped with a single-purpose tool and performs a simple repetitive task for years, the robot can be considered as a part of a single-purpose process and thus a machine that is not universal in its application. Based on this assumption, the article is focused on increasing task-specific repeatability.

\section{Methodology}

An industrial robot ABB IRB1200 5/0.9 [26] was chosen for the experiments. It is a compact angular six-axis robot with IP40 protection, which is designed for work in clean indoor spaces for applications such as packaging, handling, and assembly.

According to the manufacturer, the repeatability of the manipulator is $0.025 \mathrm{~mm}$, and the robot can be installed in any orientation at any angle. Table 1 contains the basic technical parameters of the robot. The forward reach of the robot is $901 \mathrm{~mm}$ with a load capacity of $5 \mathrm{~kg}$.

Table 1. Basic parameters of IRB1200 5/0.9 [26].

\begin{tabular}{cc}
\hline Position repeatability & $0.025 \mathrm{~mm}$ \\
Max. TCP speed & $8.9 \mathrm{~m} / \mathrm{s}$ \\
Max. TCP acceleration & $36 \mathrm{~m} / \mathrm{s}^{2}$ \\
Acceleration time-1 m & $0.06 \mathrm{~s}$ \\
Robot weight & $54 \mathrm{~kg}$ \\
Robot payload (max.) & $5 \mathrm{~kg}$ \\
\hline
\end{tabular}

The experiments are performed at the workstation with a pair of the ABB IRB1200 robots. The robots are mounted on a pedestal so that their bases are not in a horizontal position but are inclined by an angle of $40^{\circ}$ in the Y-axis of the world coordinate system of each of the robots (see Figure 1). This is not a standard mounting position of the robot. In this case, the first joint of the robot is loaded by torque even if it holds a position. This fact directly affects the accuracy and repeatability of the robot.

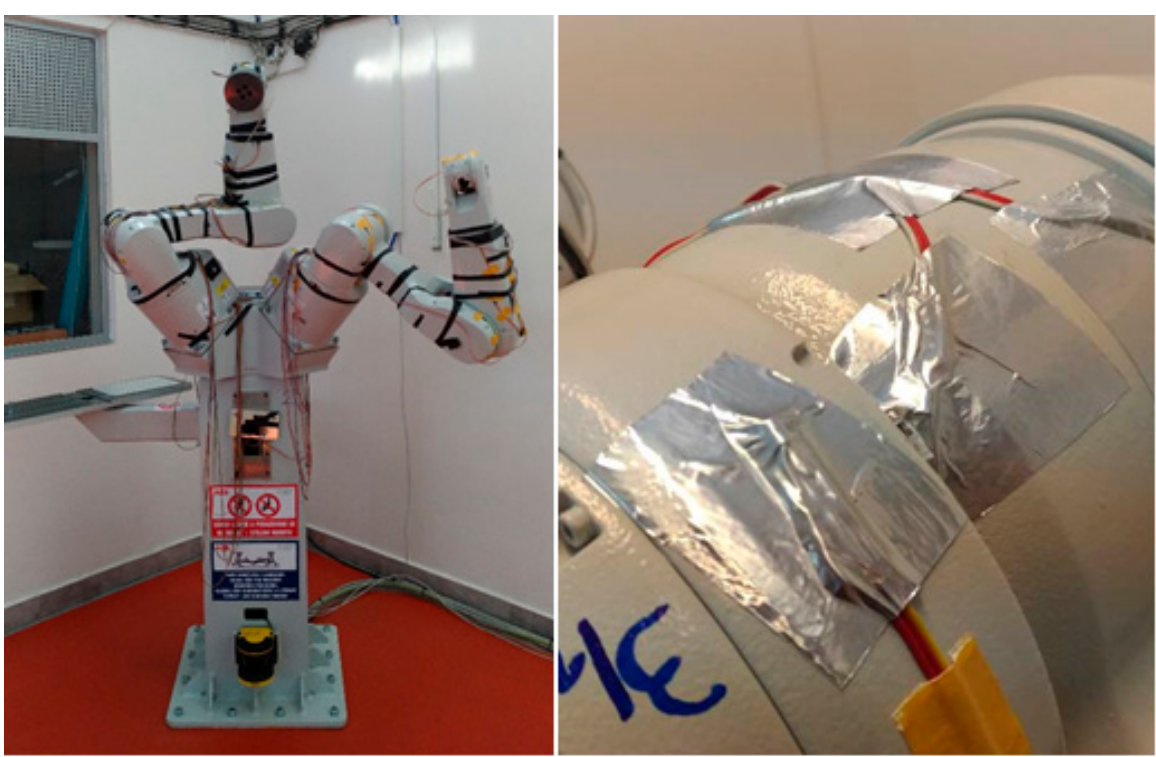

Figure 1. Thermal sensors, in detail on the second joint of the IRB1200 robot.

The minimal delay for robot stabilization is set to $0.6 \mathrm{~s}$ and was determined by the three-axis accelerometers, fixed on two places directly on the robot's flange and the base of 
the robot. This measurement was conducted during the previous research [25]. Although the arm can be considered stabilized after $0.6 \mathrm{~s}$, the delay for repeatability measurement was set to $5 \mathrm{~s}$ for higher accuracy of data obtained by the measuring system.

A temperature sensing system is necessary to ensure continuous measurement during the heating of the robot from ambient to operational temperature. The operational temperature is given, among other things, by the degree of load on individual joints during the work cycle. We performed a series of measurements for each joint to determine the optimal positions for temperature sensors. These measurements have been conducted using FLIR Ax5 thermal camera. From the images obtained from the FLIR, it is apparent that the major sources of heat are the joint gearboxes. Based on this knowledge, 25 digital temperature sensors (Dallas DS18B20) were installed, "four" sensors for each of the robot joints and "one" as an ambient temperature sensor, mounted on the pedestal of the robot. The thermal sensors were fixed by an aluminium tape (Figure 2) and for better heat distribution, a thermal compound was applied between a surface and the sensors. To verify the obtained measurement results, both workstation robots were equipped with thermal sensors.
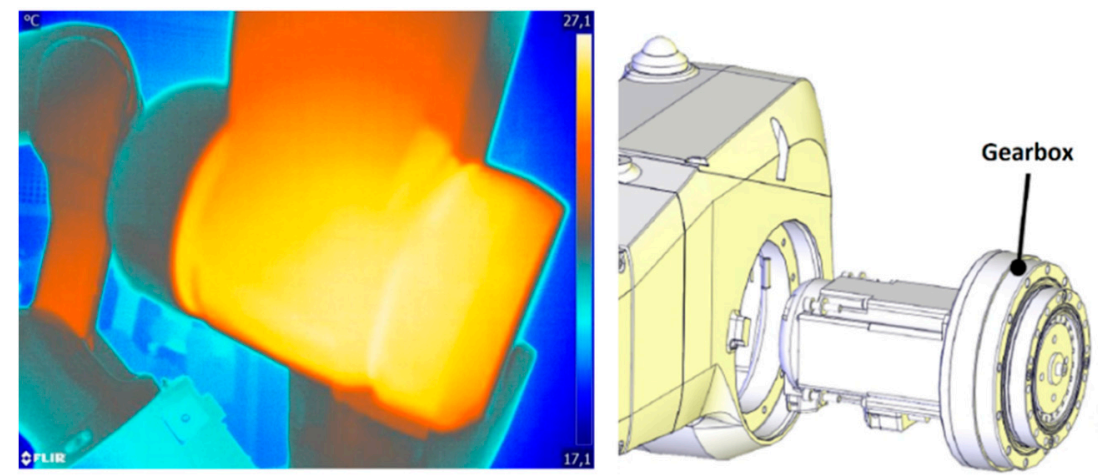

Figure 2. Heat distribution in the gearbox of the third joint of IRB1200 robot.

Data collection from these sensors is implemented via 1-Wire Bus (designed by Dallas Semiconductor) by a microprocessor system built on the ARM STM32 microprocessor platform, which pre-processes the obtained data, checks its integrity, and then sends the data via USB interface to the processing computer (Intel NUC). After processing, the data is then transferred to the robot controller via TCP/IP communication using the ABB PC SDK. Communication with displacement sensors (laser profiler and confocal sensor) is also implemented via TCP/IP interface using the appropriate libraries supplied by the manufacturer. The hardware connections are described in Figure 3.

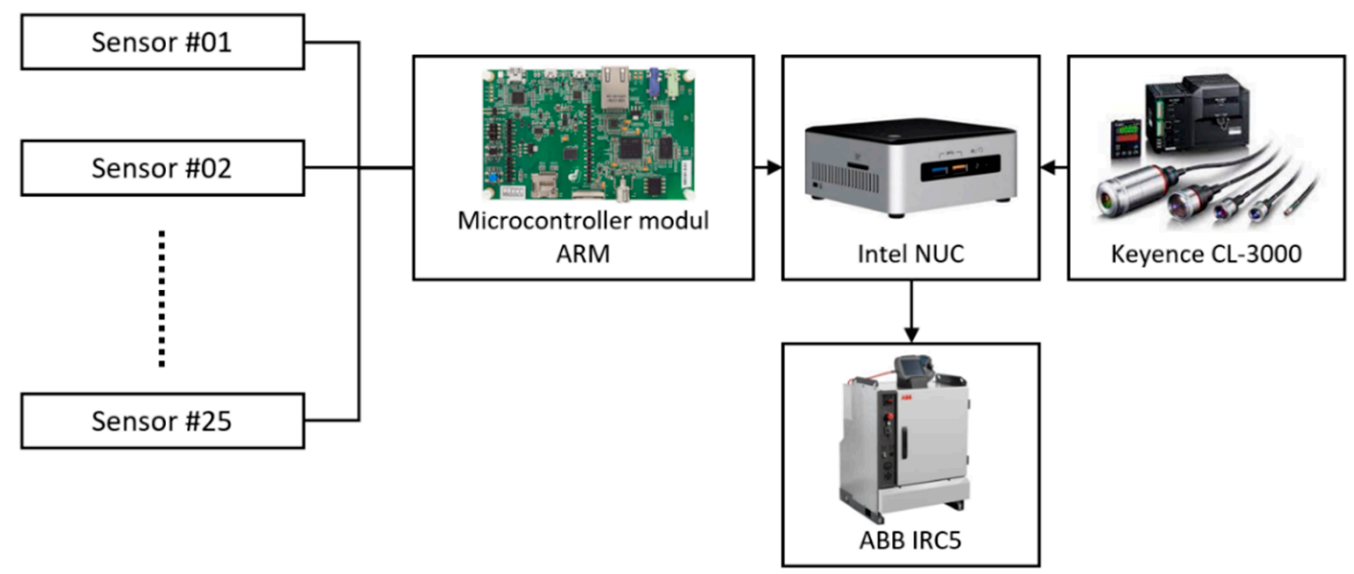

Figure 3. Block diagram of the measuring system. 
The 3 DOF repeatability is evaluated by a measuring nest (Figure 4) consisting of a Keyence CL-3000 confocal sensor unit and a pair of Keyence LJ-X8080 laser profilers. The CL-3000 confocal displacement sensor with the CL-P015/L015 sensor head measures the distance to four points of the measured surface. According to the datasheet [27] precision of this sensor is $\pm 0.49 \mu \mathrm{m}$. The Keyence LJ-X8080 is a laser profiler sensor head that detects 3200 points in a single line. For distance measurement, a middle point value is used. The value is obtained with a precision of $\pm 0.5 \mu \mathrm{m}$. When the robot is in a detection range, the measurement is triggered by a control application. The measured values from a single measurement cycle are divided into six chunks; the first two (approaching the target and stabilization) and the last (departure) are excluded from processing. The result is an average from the remaining data. A value of repeatability is read from the sensors with a rate of $100 \mathrm{~ms}$, and thus after the processing, the result is an average of approximately 25 values. The data provided by the laser profilers might be affected by a random error in the form of peaks if the laser beam angle is not set correctly. To get a valid data from the laser profiler, it is necessary to set an appropriate laser beam angle that depends on a detected surface. Based on research [28], the angle to the surface was set to $-18^{\circ}$. These settings guarantee results of the finest quality. This combination of sensors is used for its high level of precision and its availability. Any other suitable high accurate sensor such as laser tracker or Digital Image Correaliton (DIC) cameras could have been used instead.

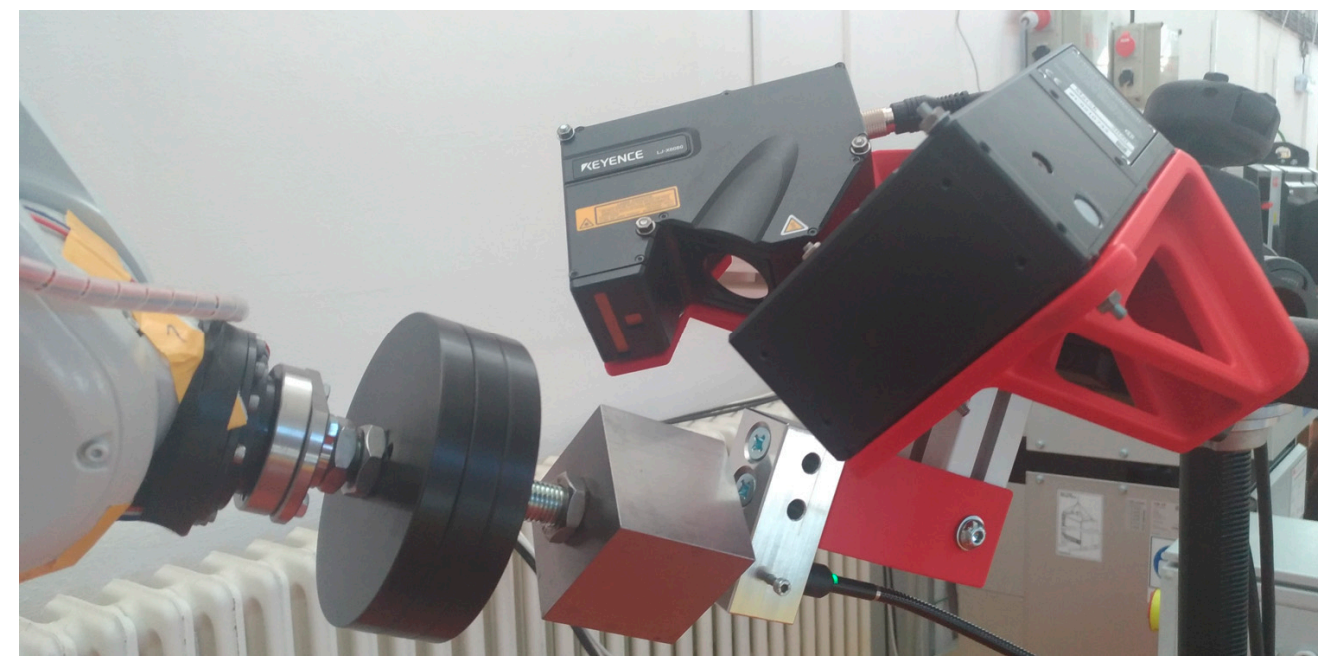

Figure 4. Measuring nest. A robot with the payload tool approaches to confocal sensor that measures the TCP Z-axis distance. TCP X and Y are measured by a pair of laser profilers above the payload tool.

All the sensors are connected via a communication network to a custom. NET C\# application that runs on Intel NUC PC. The application collects, controls, and transmits the temperature and the position deviation. In addition, it stores the data in a file for later offline analysis and, at the same time, sends the data to the robot controller for online processing utilized for a positioning error compensation (described later in this article).

\section{Data Acquisition}

This chapter describes a measurement that aims to evaluate the hypothesis that it is possible to compensate for the drift in real-time, regardless of the ambient or the robot temperature value. The aim is to increase the endpoint repeatability for the targets of interest. The authors are of the opinion that a high level of precision in industrial conditions is needed only for a small number of targets. There is usually no need to increase the robot repeatability in the whole working envelope. If the repeatability is increased for specific targets only, the achievable level of repeatability is higher than in the case of general compensation valid for a large area of the working envelope. To improve the 
repeatability by applying for the temperature-based drift compensation, we propose the following methodology.

\subsection{Warming Up Robot Joints and the Repeatability Measurement}

The first step is to obtain the relationship between the drift and the robot joint's temperature in time. The robot trajectory is defined to reach the highest possible temperature. Since the robot temperature gradually increases during recurring trajectory executions, the resulting dataset will contain data for the entire temperature range. The trajectory is defined in such a way that all joints of the robot simultaneously move between their limit positions with a defined speed. It represents the worst possible case because all the joints are moved throughout their entire working range.

A similar real-time thermal error compensation method was presented by Shibin et al. [13]. The approach utilizes the temperature of the joints; however, in contrast to our work, the researchers warmed up the joints one by one. Based on preliminary tests and measurement data (to be presented later in the text), we concluded that this solution inappropriately simplifies the result due to the fact that each joint thermally interact with other joints and are effectively cooled by the motion of the previous joints of the robot serial structure. Figure 5 describes the process of measurement. The robot executes the heating trajectory and, after every cycle, moves to the measuring nest (see Figure 4). After the stabilization delay, the output values are read from all the displacement sensors, and the cycle is repeated. When the relative joint temperatures (relative to ambient temperature) are no longer changing, the operational temperature is reached, and after an additional sixty minutes, the measurement stops.

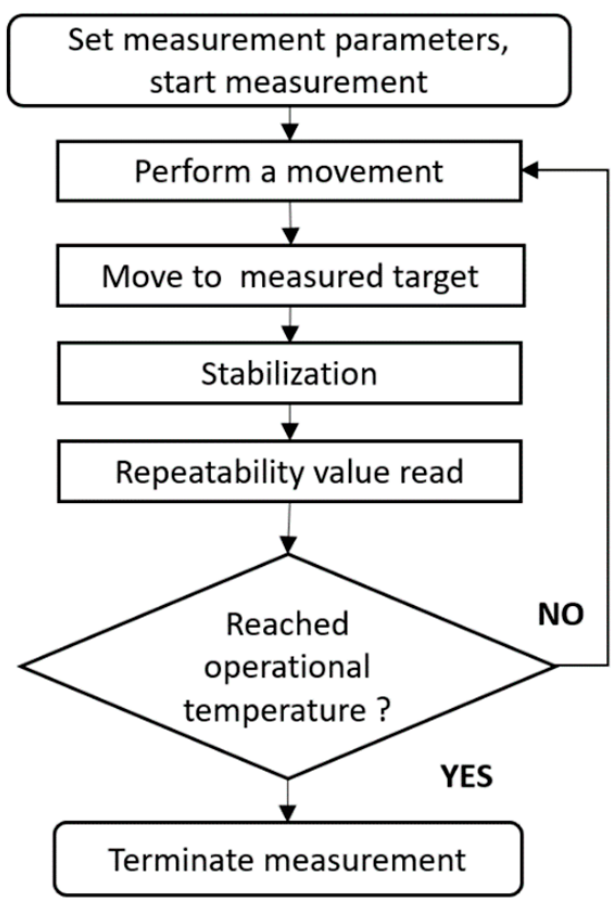

Figure 5. Flowchart of the control system for the experimental measurements.

With the use of the methodology presented in this article, the robot warm-up procedure is performed. The results of the measurements are shown in Figure 6. 


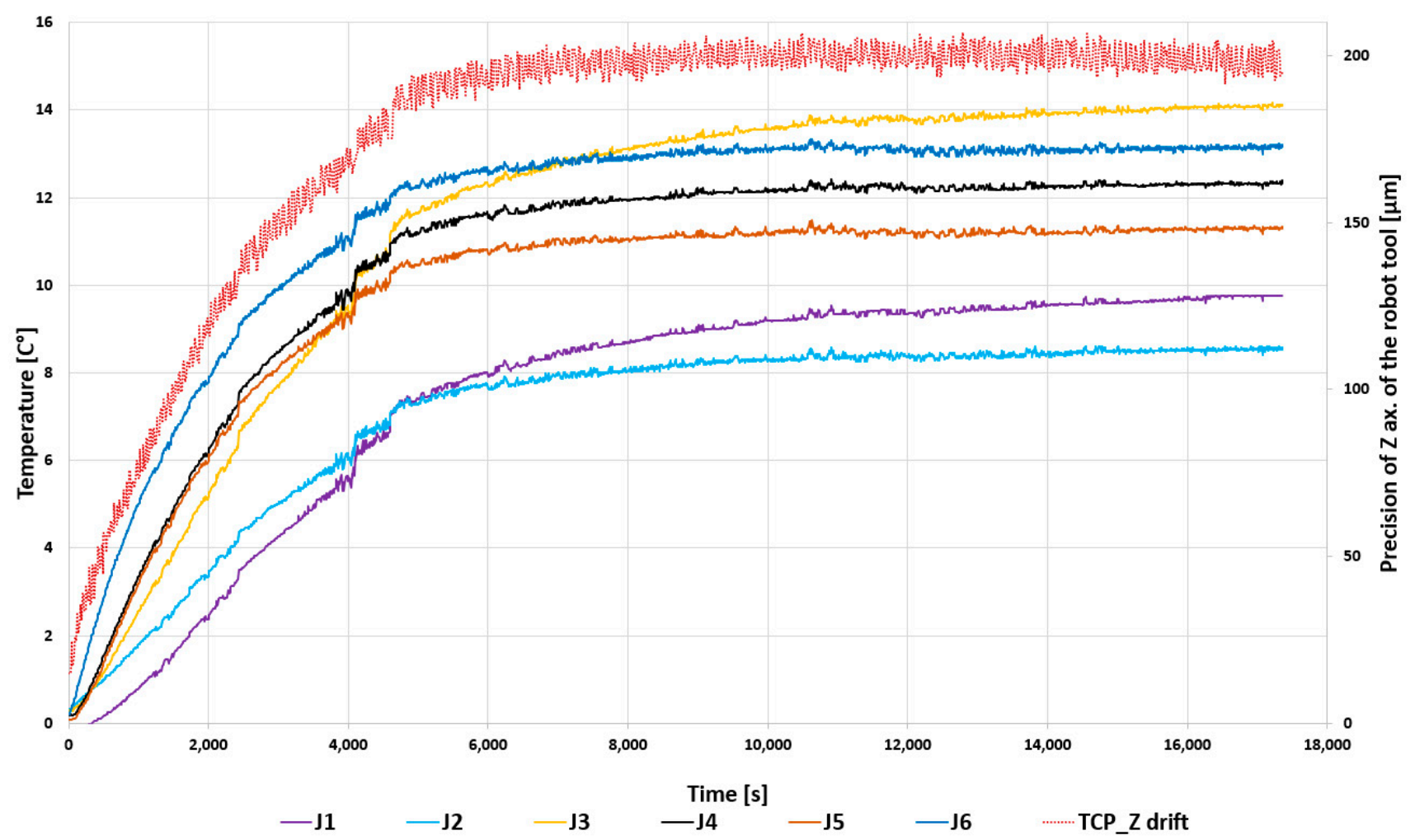

Figure 6. TCP Z-axis drift measurement, Target 1, maximum speed. Data recorded during the initial measurement: purple-joint 1 temperature, light blue-joint 2 temperature, yellow-joint 3 temperature, black-joint 4 temperature, orange-joint 5 temperature, dark blue-joint 6 temperature, red-dots express the trend of TCP Z-axis drift.

The graph (Figure 6) describes the relation between drift and joint temperatures in time. The six temperature trends displayed on the left $\mathrm{Y}$-axis of the graph visualize the average values for each robot joint (denoted by $J_{1}-J_{6}$ ). The joint average value is evaluated based on the values obtained from four sensors installed around the corresponding joint. The ambient temperature is subtracted, so the displayed values represent relative joint temperatures. The right $\mathrm{Y}$-axis of the graph describes the trend of repeatability in TCP $\mathrm{Z}$-axis. The lowest measured value is set as reference value in order to be able to compare measurement results between confocal sensor and laser profilers.

For the measurement depicted in Figure 6, the difference of the repeatability between cold and warmed-up conditions is $210 \mu \mathrm{m}$. For comparison, the robot manufacturer declares $25 \mu \mathrm{m}$ as the value of repeatability [26]; this value is, however, valid for constant temperature conditions only.

\subsection{Drift Compensation Calculation}

The compensation equation, which predicts the TCP position error based on the current temperature of the robot joints, is implemented and tested using Matlab Regression Learner. Seven inputs are defined for this prediction, each representing the average temperature of the robot's joints and the ambient temperature. The application output is defined as the prediction of the positioning errors for individual TCP axes. Figure 7 presents the single-axis output of the regression model (yellow points) and the originally measured drift (blue points). The temperature-based position error prediction corresponds to the actually measured position error. 


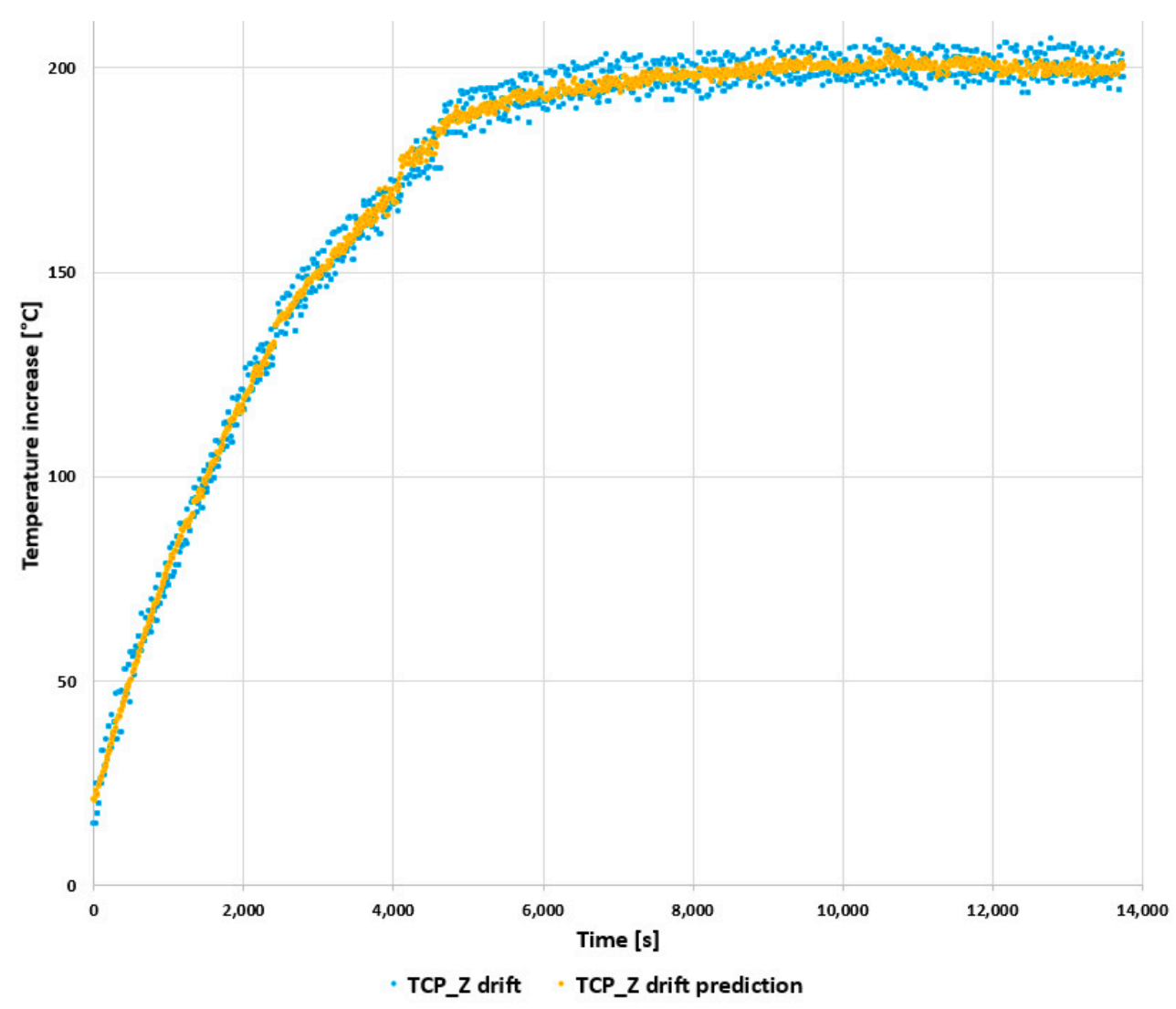

Figure 7. MatLab TCP Z Drift prediction, Target 1, maximal speed. Presented trends: blue points—-measured robot drift, yellow points—drift prediction calculated by MatLab regression learner.

After the created regression model is exported from Regression Learner to MatLab, the coefficients for the calculation can be extracted. An equation was then implemented in the form of the robot's RAPID program running in a parallel task. The task is configured to recalculate the drift compensation once per second based on all twenty-five input temperatures measured at the joints.

The final equation represented by the program in the robot controller is then:

$$
n_{Z}=\left(I+A \times T+\overline{n J_{1}} \times J_{1}+\overline{n J_{2}} \times J_{2}+\overline{n J_{3}} \times J_{3}+\overline{n J_{4}} \times J_{4}+\overline{n J_{5}} \times J_{5}+\overline{n J_{6}} \times J_{6}\right)-m .
$$

$T\{0.25\}$ input array consists of all the values from the temperature sensors. The 25th value represents the ambient temperature. The value of intercept $I$, Ambient $A$ and $J_{1}-J_{6}$ are constants given by the regression learner (see Table 2). $\overline{n J_{1}}-\overline{n J_{6}}$ are the average values of the joint temperatures (since each joint is equipped with four temperature sensors). The value of $m$ is the maximum value of the drift. This calculation has to be performed three times-for each TCP-axis separately.

Table 2. Regression coefficients.

\begin{tabular}{cc}
\hline Value for: & Estimation $[-]$ \\
\hline$I$ [Intercept] & -407.3077 \\
$A$ [Ambient] & -0.1637 \\
$J_{1}$ & 2.9261 \\
$J_{2}$ & 9.7893 \\
$J_{3}$ & -9.3647 \\
$J_{4}$ & 9.0107 \\
$J_{5}$ & 2.3683 \\
$J_{6}$ & 4.4422 \\
\hline
\end{tabular}




\subsection{Evaluation of the Drift Compensation}

The use of this technique allows to almost completely neutralize the effect of drift, as can be seen on the graph (Figure 8). Without drift compensation, the repeatability is in $210 \mu \mathrm{m}$ range (according to the trend of the TCP $\mathrm{Z}$ axis drift in the graph). When applying the calculated drift compensations based on the current robot temperature, the resulting repeatability is in $18 \mu \mathrm{m}$ range (the value is represented by the green line in Figure 8). For these specific conditions, an increase in repeatability of one order of magnitude was achieved.

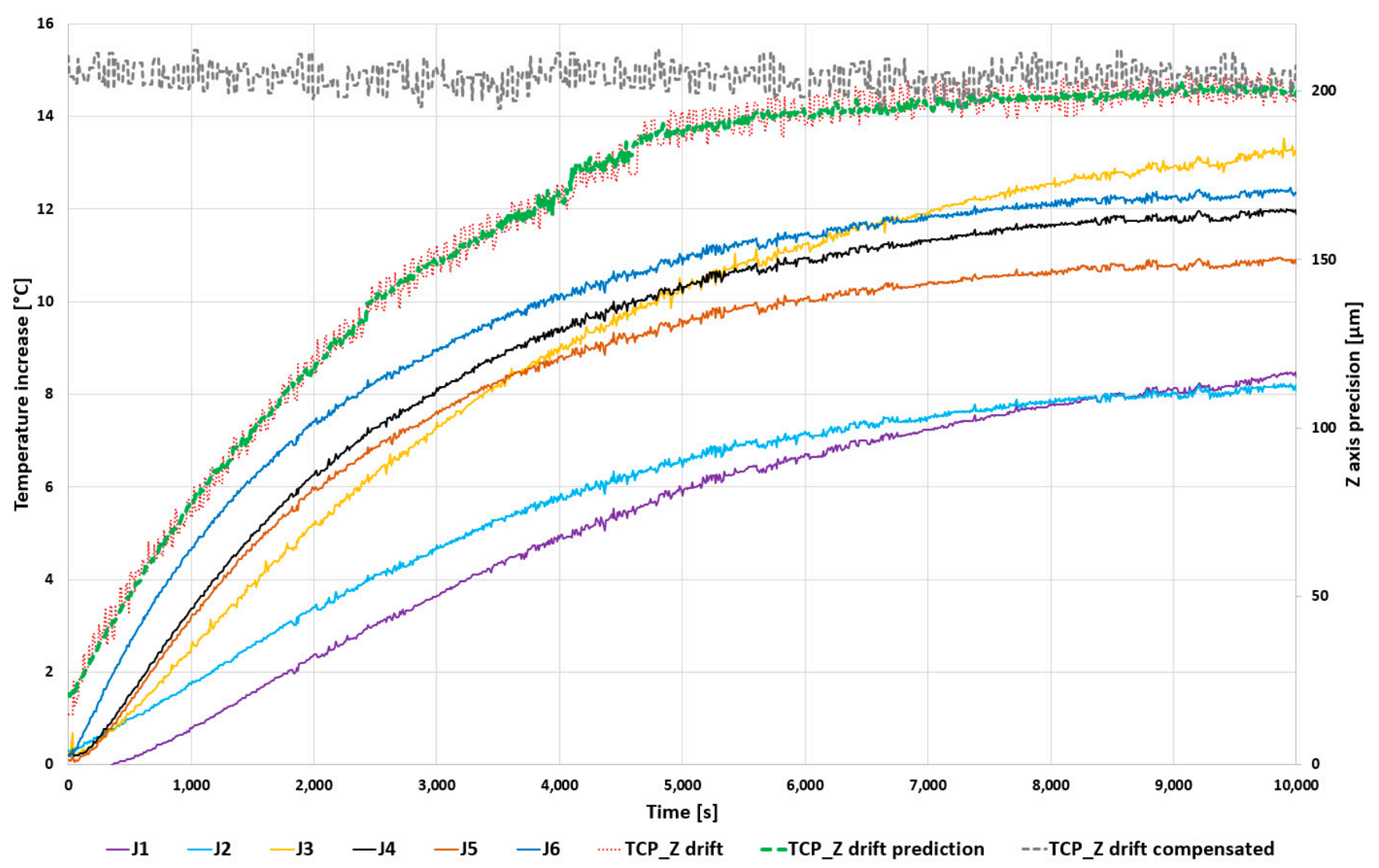

Figure 8. With and without drift compensation, Target 1, maximal speed. Data recorded during the initial measurement: purple-joint 1 temperature, light blue-joint 2 temperature, yellow-joint 3 temperature, black-joint 4 temperature, orange-joint 5 temperature, dark blue-joint 6 temperature, red dots express the uncompensated TCP Z-axis drift, green dots-TCP Z drift prediction based on MatLab regression learner and finally grey trend represents the TCP Z-axis drift with drift compensation applied.

\section{Results and Discussion}

Table 3 describes the measured targets with respect to the robot world coordinate system. The last four measured targets represent the vertices of the ISO9283 cube [1]. The measurements were performed under different conditions, such as different TCP speeds ( $300 \mathrm{~mm} / \mathrm{s}$ of working speed or $8900 \mathrm{~mm} / \mathrm{s}$ of maximal speed). Some of the measurements were performed to prove that the methodology is stable and effective even when the system is interrupted during the process. To check whether the system can handle random faults, additional measurements were performed with a delay between the cycles. 
Table 3. Target positions [mm].

\begin{tabular}{cccc}
\hline Target & $\mathbf{X}$ & $\mathbf{Y}$ & $\mathbf{Z}$ \\
\hline Target 1 & 500 & 500 & 500 \\
Target 2 & 410 & 0 & 680 \\
Target 3 & 500 & 580 & 500 \\
Target 4 & 700 & 845 & 330 \\
Target 5 & 700 & 785 & 451 \\
Target 6 & 290 & 900 & 450 \\
Target 7 & 175 & 260 & 320 \\
Target 8 & -240 & 670 & 320 \\
\hline
\end{tabular}

Figure 9 shows the data of the measurement performed under similar conditions, however, the TCP speed was set to the working speed $(300 \mathrm{~mm} / \mathrm{s})$. It is apparent that lower load causes the temperature to stabilize at lower values, which leads to less deformation of the arm. According to the TCP drift trend in the Figure 9, the repeatability difference between cold and warmed robot is $168 \mu \mathrm{m}$, whereas with the compensation, it is only $17 \mu \mathrm{m}$; thus, the influence of the drift in Z-axis of the robot tool is reduced by applying the developed compensation system.

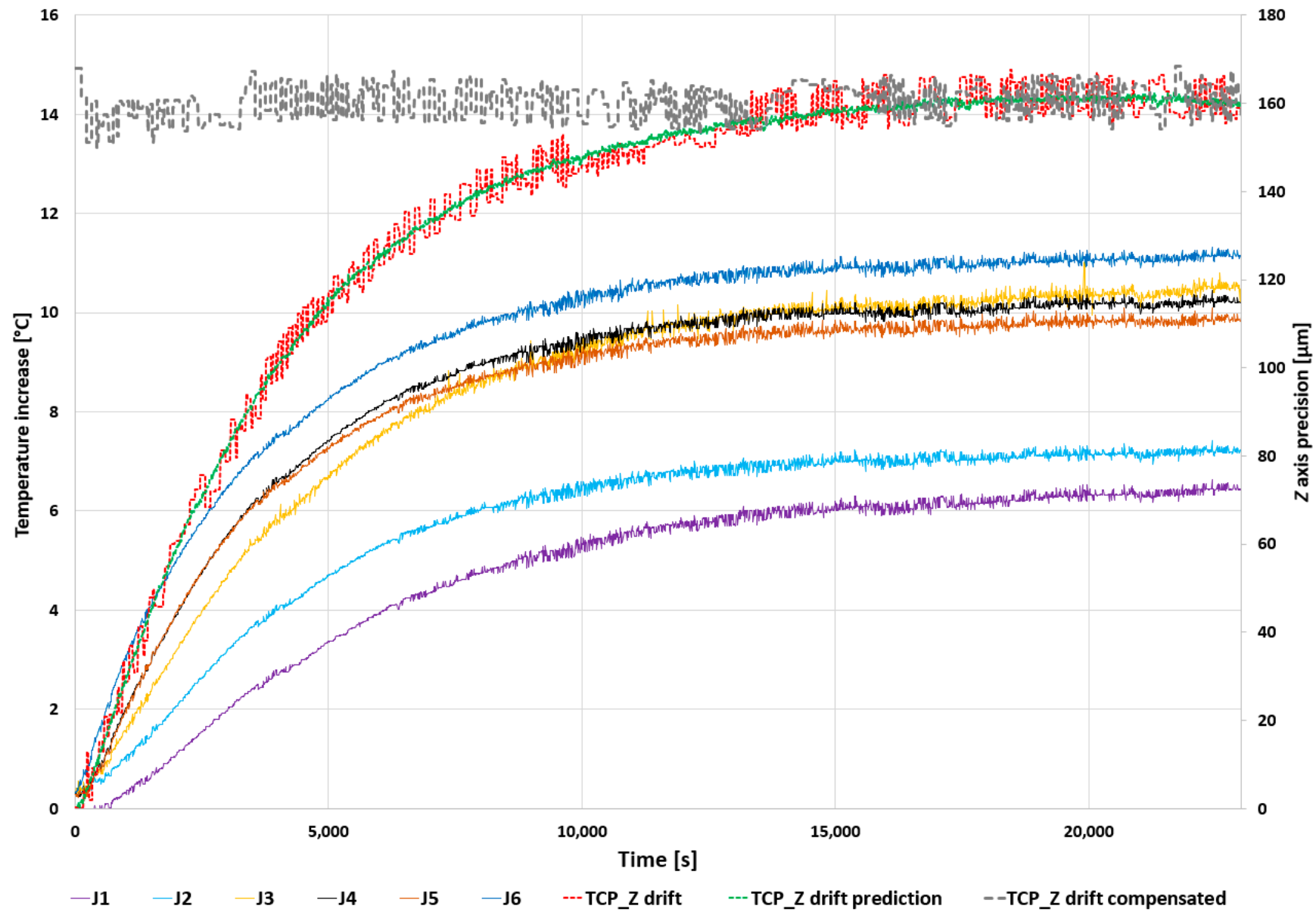

Figure 9. With and without drift compensation, Target 1, working speed. Data recorded during the initial measurement: purple-joint 1 temperature, light blue-joint 2 temperature, yellow-joint 3 temperature, black-joint 4 temperature, orange-joint 5 temperature, dark blue-joint 6 temperature, red dots express the uncompensated TCP Z-axis drift, green dots-TCP Z drift prediction based on MatLab regression learner, grey trend represents the TCP Z drift with drift compensation applied. 
The methodology efficiency was verified on multiple targets, including those defined according to the ISO 9283:1998 standard (Target 5-Target 8, see Table 2). To test the stability of the drift compensation system to external influences, the cycles with enabled compensation were deliberately interrupted by stopping the robot movement (300 s pause after every $900 \mathrm{~s}$ of movement). The result of this test is presented in Figures 10 and 11. For Target 5 (reached at working speed), the difference in repeatability is $205 \mu \mathrm{m}$-see TCP Z-axis trend in Figure 10. With the compensation system enabled, the subsequent measurement (Figure 11) was intentionally interrupted several times. As shown in Figure 11, the compensation system counterbalanced the error down to $31 \mu \mathrm{m}$ (repeatability error reduction of $84.9 \%$ ), even though the regression model was trained from the undisturbed dataset.

Figure 11 reveals an interesting phenomenon: when the robot's movement is stopped, a temperature rise over a short period of time can be observed. This phenomenon can be partially explained by a difference in the thermal properties of the materials used in the design of the robot. The primary heat sources, the gearboxes, are made of steel with a density of $7850 \mathrm{~kg} / \mathrm{m}^{3}$, but the robot links (lower and upper arm) are made of aluminium alloy with a density of $2750 \mathrm{~kg} / \mathrm{m}^{3}$. The heat accumulated in the gearboxes is dissipated in the surrounding aluminium frame, but when the manipulator is not in motion, the frame is not air-cooled, resulting in a local temperature rise on the frame. For this reason, we did not perform heating and cooling evaluation for individual joints and all the joints were measured simultaneously instead.

Taken together, the results indicate that the presented compensation system can significantly reduce the effect of the drift. Unlike previous graphs, which presented results for a single TCP-axis, Figure 12 presents the drift difference between non-compensated and compensated robot motion for all TCP axes. The significant increase of repeatability is especially noticeable for the TCP Z-axis (the red line of the graph), however, $\mathrm{X}$ and Y-axis are also suitable to compensate, as their differences in repeatability vary considerably.

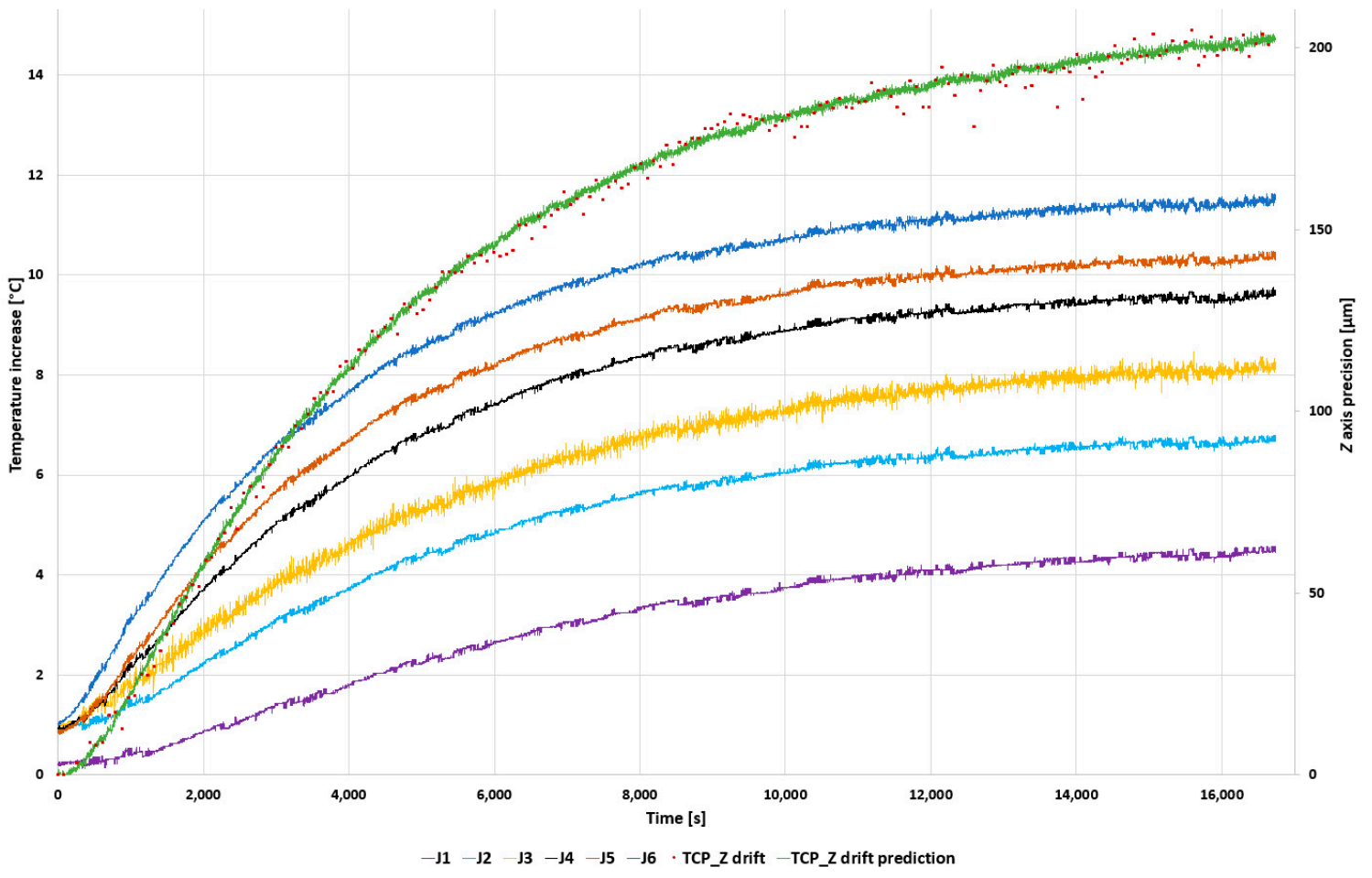

Figure 10. Target 5, working speed TCP Z Drift measurement and prediction. Data recorded during the initial measurement: purple-joint 1 temperature, light blue-joint 2 temperature, yellow-joint 3 temperature, black-joint 4 temperature, orange-joint 5 temperature, dark blue-joint 6 temperature, red dots express the uncompensated TCP Z-axis drift, green dots-TCP Z drift prediction based on MatLab regression learner. 


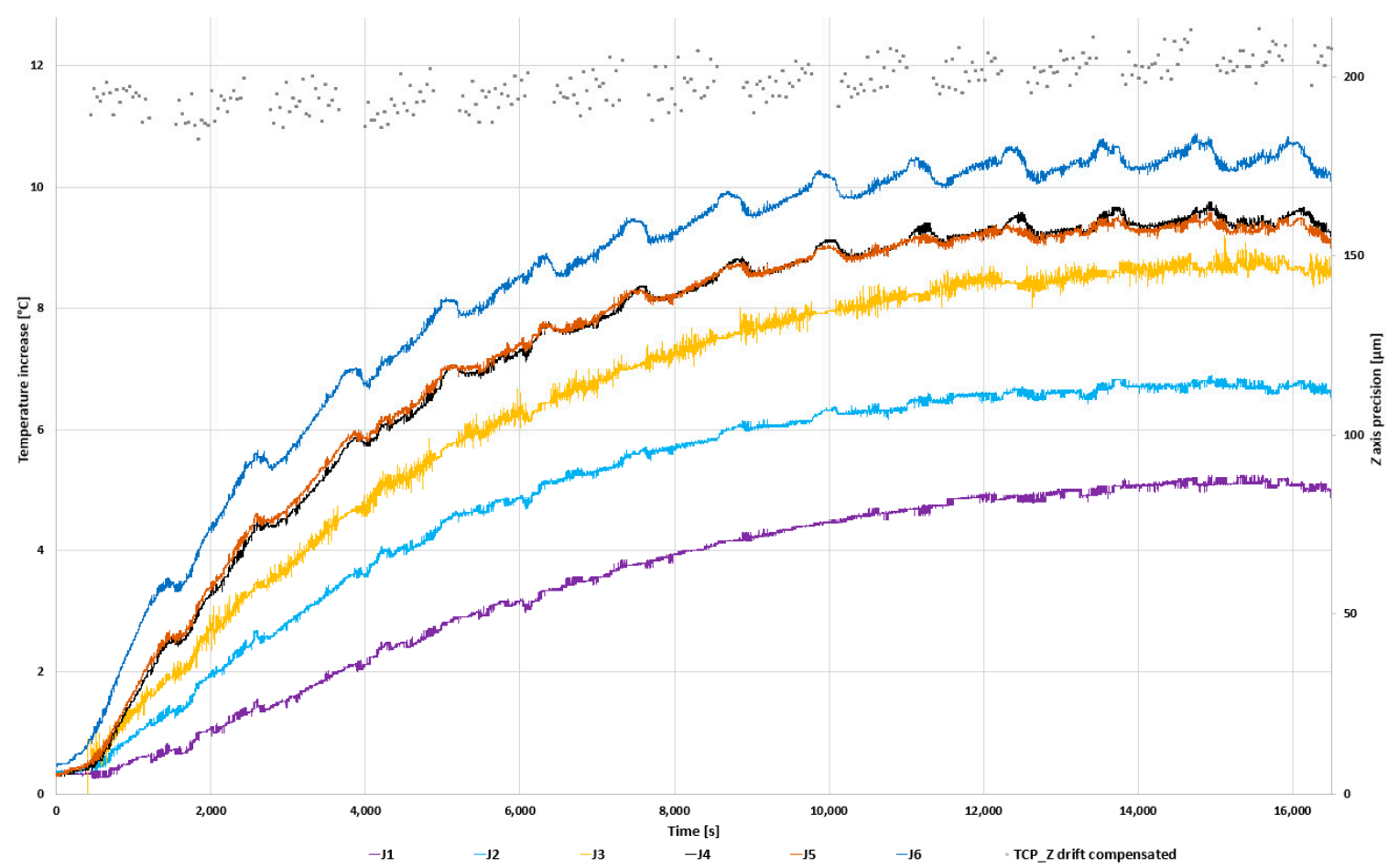

Figure 11. Target 5, working speed, compensated TCP Z Drift, measurement with interrupts. Data recorded during the initial measurement: purple-joint 1 temperature, light blue-joint 2 temperature, yellow-joint 3 temperature, black-joint 4 temperature, orange- joint 5 temperature, dark blue-joint 6 temperature, grey trend represents the TCP Z drift with drift compensation applied on measurement that was systematically disrupted.

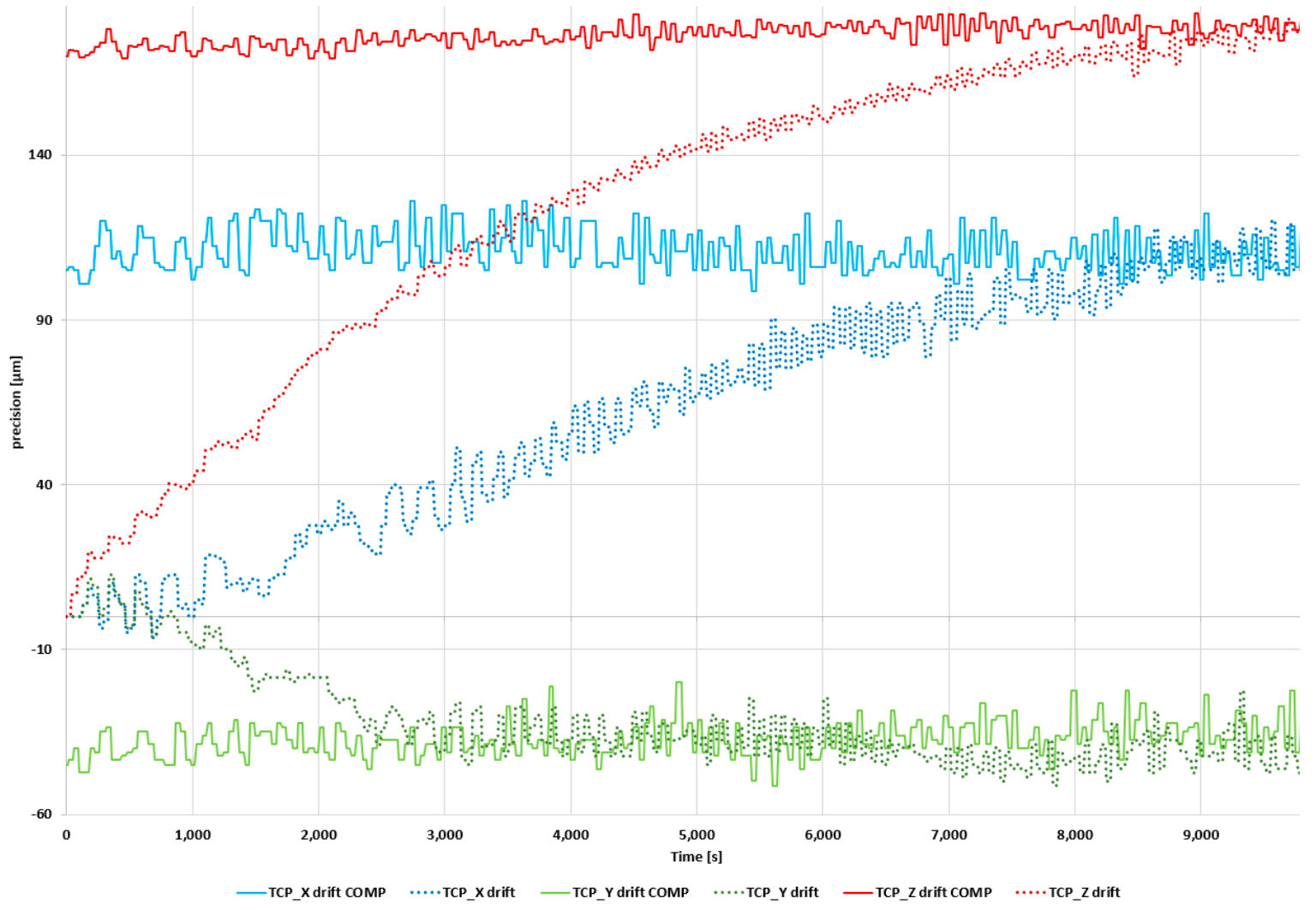

Figure 12. Target 5, working speed, 3DOF repeatability comparison. Blue dots-TCP X not compensated, blue line-TCP X compensated, green dots-TCP Y not compensated, green line-TCP Y compensated, red dots-TCP Z not compensated, red line-TCP Z compensated. 
After evaluating all measurements and considering the presented results, the authors consider the proposed methodology to be fully functional. Application of this methodology allows compensating for the effect of temperature on TCP repeatability.

\section{Conclusions}

The results of the presented measurements indicate the usefulness of the developed compensation methodology since it significantly improves repeatability. When compensated, the value of the positioning error is stabilized, and the influence of the drift, caused by the structural thermal expansion, is reduced. The hypothesis that the repeatability error caused by the effect of structural thermal expansion can be reduced and the repeatability values guaranteed for a steady temperature can be achieved even during the heating of the robot to operating temperatures, was proved.

For all the measurements performed, the repeatability with applied drift compensation is approximately $25 \mu \mathrm{m}$ at any temperature. Without compensation, the repeatability is usually in the range of $200 \mu \mathrm{m}$. Many effects impact the repeatability value. However, the effect of thermal expansion is the most fundamental one. By reducing this effect, the repeatability can be increased significantly. In case of measurement of the target 5-performed under working speed (see Figures 10 and 11), the Z-axis drift was reduced by $84.9 \%$. For a target 1 , working speed measurement (see Figure 9), the Z-axis drift was reduced by $89.9 \%$. To verify the results and to obtain the accurate values of robot repeatability, all measurements were performed with a highly accurate measurement system consisting of a confocal sensor and a pair of laser profilers.

This research presents the possible solution of the robot drift compensation that potentially can save tenths of minutes of production time. Figure 12 shows that it takes the robot almost 150 min to fully stabilize the temperature. Clearly, in typical industrial applications, robots will be loaded less than in the tests carried out in this study, but it will still take tens of minutes to warm up the robot structure. When using the presented technique, the robot warming up procedure may no longer be necessary, or its duration may be significantly reduced.

For high-precision robotic applications, it is necessary to consider the environment and take steps to maximize temperature stabilization, as rapid changes in ambient temperature can affect the robot even with the temperature compensation presented in this article. In general, the optimal deployment is when the robot workspace is enclosed in a separate air-conditioned chamber. In industrial applications, the robot wears out over time, leading to an increased backlash in the gears and differences in the resistance of the bearings. Therefore, it is necessary to accept that the compensation of the robot has limited validity, and after a certain time, it will be necessary to perform it again; in our case, it may be necessary to update the constants of the compensation equations. For a standard industrial robot, the compensation validity may be similar to gearbox oil service life.

\section{Future Work}

The presented methodology is intended for the drift compensation in X-, Y-, Z-axes. We further plan to apply this methodology to a collaborative UR10 robot in an object measurement application, which requires the utmost precision of the robot.

From the presented results, it is apparent that a significant reduction of the effect of the drift applies only to a single target within the robot working envelope. If a larger number of targets needs to be compensated, this methodology seems to be inappropriate due to the time-consuming determination of the compensation equations. However, if multiple targets have the same axis orientation and are at a certain (close) distance from the initial target, it can hypothesized that those could be compensated by a single set of compensation equations. We, therefore, plan a series of tests to determine the quality of compensation of other targets that will be located in a defined distance from the initial target.

Author Contributions: M.V.: software, writing-review \& editing; Z.B.: methodology, supervision, data curation, formal analysis, validation, project administration, review \& editing; J.B.: 
methodology, software; J.S.: visualization; S.G.: review \& editing, visualization; J.M.: software, review \& editing; V.K.: conceptualization, resources, funding acquisition; M.H.: software, investigation, review \& editing. All authors have read and agreed to the published version of the manuscript.

Funding: This article was supported by the project Research Centre of Advanced Mechatronic Systems, reg. No. CZ.02.1.01/0.0/0.0/16 019/0000867 in the frame of the Operational Program Research, Development and Education. This work has been also supported by specific research project SP2021/47 and financed by the state budget of the Czech Republic and VEGA 1/0500/20 of a Slovak Grant Agency.

Institutional Review Board Statement: Not applicable.

Informed Consent Statement: Not applicable.

Data Availability Statement: Contact corresponding author.

Conflicts of Interest: The authors declare no conflict of interest.

\section{References}

1. $\quad$ ISO 9283:1998. Manipulating Industrial Robots: Performance Criteria and Related Test Methods. 2; ISO: Geneva, Switzerland, 1998 ; p. 4.

2. AccuBeam: Robot Temperature Compensation \& TCP/Robot Mastering Recovery System; DYNALOG: Bloomfield Hills, MI, USA, 2019; Available online: https:/ / www.dynalog-us.com/accubeam-robot-temperature-compensation-system.htm (accessed on 28 September 2019).

3. Temperature Compensation; WIEST AG-Kalibriersysteme: Neusäß, Germany, 2019; Available online: https://www.wiest-ag.de/ en/temperaturkompensation.html (accessed on 28 September 2019).

4. Lubrano, E.; Clavel, R. Thermal Calibration of a 3 DOF Ultra High-Precision Robot Operating in Industrial Environment. In Proceedings of the 2010 IEEE International Conference on Robotics and Automation, Anchorage, AK, USA, 3-7 May 2010; IEEE: Piscataway, NJ, USA, 2010; pp. 3692-3697, ISBN 978-1-4244-5038-1. [CrossRef]

5. MAREŠ, M.; Otakar, H.; Lukáš, H. Thermal error compensation of a 5-axis machine tool using indigenous temperature sensors and CNC integrated Python code validated with a machined test piece. Precis. Eng. 2020, 66, 21-30. [CrossRef]

6. Elatta, A.Y.; Li, P.G.; Fan, L.Z.; Yu, D. An Overview of Robot Calibration. Inf. Technol. J. 2003, 3, 74-78. [CrossRef]

7. Li, R.; Zhao, Y. Dynamic error compensation for industrial robot based on thermal effect model. Measurement 2016, 88, 113-120. [CrossRef]

8. Niu, B. Enhanced Robot Calibration by Minimization of TCP Drifts during Reorientation. In Proceedings of the 2018 IEEE/ASME International Conference on Advanced Intelligent Mechatronics (AIM), Auckland, New Zealand, 9-12 July 2018; pp. 69-74, ISBN 978-1-5386-1854-7. [CrossRef]

9. Jeon, J.W.; Park, S.-H.; Kim, S. Compensation for servo drift in industrial robots. In Proceedings of the First IEEE Conference on Control Applications, Dayton, OH, USA, 13-16 September 1992; pp. 318-323. [CrossRef]

10. Abdulshahed, A.; Abdulshahed, A.; Longstaff, A.P.; Fletcher, S.; Myers, A. Application of GNNMCI(1, N) to environmental thermal error modelling of CNC machine tools. In The 3rd International Conference on Advanced Manufacturing Engineering and Technologies; KTH Royal Institute of Technology: Stockholm, Sweden, 2013; pp. 253-262. [CrossRef]

11. Wang, D.; Bai, Y.; Zhao, J. Robot manipulator calibration using neural network and a camera-based measurement system. Trans. Inst. Meas. Control 2012, 34, 105-121. [CrossRef]

12. Olabi, A.; Damak, M.; Bearee, R.; Gibaru, O.; Leleu, S. Improving the accuracy of industrial robots by offline compensation of joints errors. In Proceedings of the 2012 IEEE International Conference on Industrial Technology, Athens, Greece, 19-21 March 2012; pp. 492-497. [CrossRef]

13. Yin, S.; Guo, Y.; Ren, Y.; Zhu, J.; Yang, S.; Ye, S. Real-time thermal error compensation method for robotic visual inspection system. Int. J. Adv. Manuf. Technol. 2014, 75, 933-946. [CrossRef]

14. Kluz, R.; Trzepieciński, T. The repeatability positioning analysis of the industrial robot arm. Assem. Autom. 2014, 34, 285-295. [CrossRef]

15. Liu, B.; Zhang, F.; Qu, X. A Method for Improving the Pose Accuracy of a Robot Manipulator Based on Multi-Sensor Combined Measurement and Data Fusion. Sensors 2015, 15, 7933-7952. [CrossRef] [PubMed]

16. Huczala, D.; Oščádal, P.; Spurný, T.; Vysocký, A.; Vocetka, M.; Bobovský, Z. Camera-Based Method for Identification of the Layout of a Robotic Workcell. Appl. Sci. 2020, 10, 7679. [CrossRef]

17. Oščádal, P.; Heczko, D.; Vysocký, A.; Mlotek, J.; Novák, P.; Virgala, I.; Sukop, M.; Bobovský, Z. Improved Pose Estimation of Aruco Tags Using a Novel 3D Placement Strategy. Sensors 2020, 20, 4825. [CrossRef] [PubMed]

18. Kluz, R.; Kubit, A.; Sep, J.; Trzepiecinski, T. Effect of temperature variation on repeatability positioning of a robot when assembling parts with cylindrical surfaces. Eksploat. I Niezawodn.-Maint. Reliab. 2018, 20, 503-513. [CrossRef]

19. Chen, D.; Yuan, P.; Wang, T.; Cai, Y.; Xue, L. A Compensation Method for Enhancing Aviation Drilling Robot Accuracy Based on Co-Kriging. Int. J. Precis. Eng. Manuf. 2018, 19, 1133-1142. [CrossRef] 
20. Moeller, C.; Schmidt, H.C.; Koch, P.; Boehlmann, C.; Kothe, S.; Wollnack, J.; Hintze, W. Real Time Pose Control of an Industrial Robotic System for Machining of Large Scale Components in Aerospace Industry Using Laser Tracker System. SAE Int. J. Aerosp. 2017, 10, 100-108. [CrossRef]

21. Kluz, R.; Kubit, A.; Trzepiecinski, T. Investigations of temperature-induced errors in positioning of an industrial robot arm. J. Mech. Sci. Technol. 2018, 32, 5421-5432. [CrossRef]

22. Heisel, U.; Richter, F.; Wurst, K.H. Thermal Behaviour of Industrial Robots and Possibilities for Error Compensation. CIRP Ann. 1997, 46, 283-286. [CrossRef]

23. Mohnke, C.; Reinkober, S.; Uhlmann, E. Constructive methods to reduce thermal influences on the accuracy of industrial robots. Procedia Manuf. 2019, 33, 19-26. [CrossRef]

24. Ristea, A.; Aurel, O. Temperature Effects on Performance of Industrial Robots Made of Composite Materials. Adv. Mater. Res. 2011, 403, 603-606. [CrossRef]

25. Vocetka, M.; Huňady, R.; Hagara, M.; Bobovský, Z.; Kot, T.; Krys, V. Influence of the Approach Direction on the Repeatability of an Industrial Robot. Appl. Sci. 2020, 10, 8714. [CrossRef]

26. Technical Data for the IRB 1200 Industrial Robot. Available online: https://new.abb.com/products/robotics/industrial-robots/ irb-1200/irb-1200-data (accessed on 26 November 2019).

27. 2D/3D Laser Profiler: LJ-X8000 Series; Keyence: Milton Keynes, UK, 2021; Available online: https://www.keyence.co.uk/products / measure/laser-2d/lj-x8000/models/lj-x8080/ (accessed on 4 October 2021).

28. Heczko, D.; Oščádal, P.; Kot, T.; Huczala, D.; Semjon, J.; Bobovský, Z. Increasing the Reliability of Data Collection of Laser Line Triangulation Sensor by Proper Placement of the Sensor. Sensors 2021, 21, 2890. [CrossRef] [PubMed] 